\title{
PREDICTORES CLÍNICOS DE TUBERCULOSIS MULTIDROGORRESISTENTE EN PACIENTES CON TUBERCULOSIS PULMONAR EN HUÁNUCO, PERÚ. 2010-2015
}

\author{
PREDICTOR FACTORS FOR MULTIDRUG-RESISTANT TUBERCULOSIS AMONG PATIENTS WITH \\ PULMONARY TUBERCULOSIS IN HUÁNUCO, PERU. 2010-2015 \\ Anghella Valdivia-Gómez ' ,Noelia Zavala-Lazo ${ }^{1}$, Bernardo Dámaso-Mata ${ }^{1,2}$, Vicky Panduro-Correa ${ }^{1,3}$, \\ Edinho Segama-Fabian ${ }^{4}$, Kovy Arteaga-Livias ${ }^{1,2}$
}

\begin{abstract}
RESUMEN
Introducción: En la ciudad de Huánuco se observa un crecimiento progresivo de casos de tuberculosis y cada vez más frecuente la tuberculosis multidrogorresistente, por esta razón fue necesario identificar factores predictores para desarrollar tuberculosis multidrogorresistente. Objetivo: Determinar si la fiebre persistente, el patrón radiográfico cavitario y la baciloscopía positiva al primer mes de tratamiento son predictores de tuberculosis multidrogorresistente en pacientes con tuberculosis Pulmonar en cuatro establecimientos de salud de la ciudad de Huánuco, entre enero 2010 y diciembre 2015. Métodos: Estudio observacional, casos y controles. Se consideraron 37 casos y 111 controles. Los datos se recolectaron de las historias clínicas. Resultados: Los factores predictores encontrados en el análisis bivariado fueron la persistencia de la fiebre a las dos semanas ( $p=0,001 ; O R \quad 0,05 ; \mathrm{IC} 0,01-0,5)$, el patrón radiográfico cavitario ( $p=0,000$; OR 11,6; IC 4,6-26,5), y la positividad de la baciloscopía al primer mes de tratamiento ( $p=0,00 ;$ OR 13,5; IC 4,1-44,6 al 95\%). Estas fueron confirmadas con el análisis multivariado ( $p=0,08 ; p=0,002 ; p=0,00$ respectivamente). Conclusión: La persistencia de la fiebre a las dos semanas, el patrón radiográfico cavitario y la baciloscopía positiva al primer mes de tratamiento mostraron asociación predictiva en forma independiente para el diagnóstico de tuberculosis multidrogorresistente.
\end{abstract}

Palabras clave: Tuberculosis; Tuberculosis farmacoresistente; Estudios de casos y controles (fuente: DeCS BIREME).

\begin{abstract}
Introduction: In the city of Huánuco and around the world, there is a progressive increase in cases of tuberculosis and multidrug-resistant tuberculosis, for this reason it was necessary to identify predictors to develop multidrug-resistant tuberculosis. Objective: To determine if persistent fever, cavitary radiographic pattern and positive smear microscopy at the first month of treatment, are predictors of multidrug-resistant tuberculosis in patients with pulmonary tuberculosis, in four health facilities in the city of Huánuco, between January 2010 and December 2015. Methods: Case and control study. We considered 37 cases and 111 controls. Data were collected from medical records. Results: The predictive factors found in the bivariate analysis were persistence of fever at 2 weeks ( $p=0.001$, OR $0.05, \mathrm{Cl} 0.01-0.5)$, cavitary radiographic pattern $(p=0.000$, OR $11.6,95 \% \mathrm{IC} 4.6-26.5)$, and smear microscopy positivity at the first month of treatment $(p=$ $0.00, \mathrm{OR} 13.5,95 \% \mathrm{Cl} 4.1-44.6)$. These were confirmed by multivariate analysis $(\mathrm{p}=0.012, \mathrm{p}=0.00, \mathrm{p}=0.00$ respectively). Conclusion: The persistence of fever at two weeks, cavitary radiographic pattern and positive smear microscopy at the first month of treatment were predictive factors associated independently for the diagnosis of multidrug-resistant Tuberculosis.
\end{abstract}

Key words: Tuberculosis; Multidrug - resistant tuberculosis; Case and control studies (source: MeSH NLM).

\footnotetext{
${ }^{1}$ Facultad de Medicina. Universidad Nacional Hermilio Valdizán, Huánuco-Perú.

${ }^{2}$ Hospital II EsSalud, Huánuco-Perú.

${ }^{3}$ Hospital Regional Hermilio Valdizán, Huánuco-Perú.

${ }^{4}$ Hospital Nacional Ramiro Prialé, Huancayo-Perú.

Citar como: Anghella Valdivia-Gómez,Noelia Zavala-Lazo, Bernardo Dámaso-Mata, Vicky Panduro-Correa, Edinho Segama-Fabian, Kovy ArteagaLivias. Predictores clínicos de tuberculosis multidrogorresistente en pacientes con tuberculosis pulmonar en Huánuco, Perú. 2010-2015 Rev. Fac. Med. Hum. Abril 2020; 20(2):193-200. DOI 10.25176/RFMH.v20i2.2711
} 


\section{INTRODUCCIÓN}

La tuberculosis multidrogorresistente (TB-MDR) se define como la resistencia simultánea a isoniacida y rifampicina, siendo considerado en la actualidad un importante problema de salud pública, principalmente en los países en vías de desarrollo, donde existen altos índices de pobreza ${ }^{(1)}$.

Según la OMS, en el año 2016 aproximadamente 490 000 personas en el mundo desarrollaron TB-MDR, de estos pacientes, casi el 50\% de ellos se encontraban en la India, la República Popular de China y la Federación de Rusia ${ }^{(1)}$. En los países de américa la tasa general de casos nuevos en el año 2011 fue de 28 por 100000 habitantes, siendo los países con mayor incidencia Haití y Bolivia; sin embargo, los casos notificados por Brasil y Perú representaban más de la mitad de todos los casos en la región ${ }^{(2)}$. En el Perú, según los datos publicados por el Ministerio de Salud y la OMS, el 5\% de todos los casos de tuberculosis en el Perú son TB-MDR y el $82 \%$ de éstos se concentran en las zonas de Lima y Callao ${ }^{(3,4)}$. En el departamento de Huánuco, durante el año 2014 se registraron 22 casos nuevos de TB-MDR confirmada, de los cuales el 59\% correspondieron a la provincia de Huánuco, siendo la ciudad de Huánuco donde se concentran la mayor parte de casos de TB-MDR ${ }^{(5)}$.

Las pruebas de sensibilidad, tanto moleculares y de cultivos, son los marcadores esenciales para el diagnóstico y manejo adecuado de la TB-MDR; lamentablemente algunas de estas pruebas son costosas, otras de resultados tardíos y no disponibles universalmente, sobre todo en áreas con recursos limitados como Huánuco, que se encuentra ubicado en la zona centro oriental del Perú, es la segunda región más pobre del Perú con un 30\% de personas en extrema pobreza, la cuarta parte de su población no tiene el servicio de desagüe, el 9,2\% de los habitantes no cuenta con el acceso al agua potable, y donde actualmente se concentra una importante carga de incidencia de casos de tuberculosis; por lo que es imperativo desarrollar nuevas metodologías, más rápidas y asequibles para facilitar la identificación de pacientes con TB-MDR ${ }^{(6)}$.

En este contexto, mundialmente se han llevado a cabo una serie de estudios utilizando la clínica, la radiología, los exámenes de laboratorio y hasta la inmunogenética, con el objetivo de establecer factores predictores de TBMDR, entre cuyos resultados destacan la asociación con infección por VIH, la presencia de adenopatías hiliares o mediastinales en la radiografía de tórax y la ausencia de infiltrados en la radiografía de tórax ${ }^{(7)}$; del mismo modo otros factores asociados son ser joven, abandonar el tratamiento y la presencia de lesiones cavitarias en la radiografía de tórax ${ }^{(8)}$; mientras que en el Perú otro estudio encontró que el contacto con un paciente con TB-MDR dentro de la familia, un examen pulmonar anormal y el patrón cavitario en las radiografías de tórax son factores relacionados a presentar TB-MDR ${ }^{(6)}$.

Bajo estas consideraciones, podemos observar cuán importante es identificar factores predictores en la TBMDR que puedan ser accesibles en nuestra realidad, siendo esto necesario para realizar una búsqueda activa y razonada de pacientes con potencial riesgo, disminuir el tiempo de diagnóstico y ofrecer un tratamiento adecuado y oportuno; logrando de esta manera un mejor control de la tuberculosis, evitando la transmisión de la enfermedad y consecuentemente proporcionar una adecuada prevención secundaria. Todas estas acciones tendrán mayor impacto en la población de menos recursos económicos, quiénes son los más vulnerables a esta enfermedad en Huánuco, el Perú y otras partes del mundo ${ }^{(9,10)}$.

En tal escenario, los objetivos de nuestro estudio fueron establecer factores predictores para desarrollar TBMDR a partir de parámetros clínicos, radiológicos y de laboratorio de fácil acceso en áreas de recurso limitados.

\section{MÉTODOS}

\section{Diseño}

Se realizó un estudio observacional, analítico, longitudinal, retrospectivo, caso-control entre enero de 2010 y diciembre de 2015; el cual incluyó registros de pacientes con tuberculosis pulmonar de cuatro establecimientos de salud de la ciudad de Huánuco: centro de salud (CS) Aparicio Pomares, CS Las Moras, hospital II EsSalud Huánuco y el hospital regional Hermilio Valdizán.

\section{Población y muestra}

Durante el periodo de estudio se encontraron 200 historias clínicas de pacientes con tuberculosis pulmonar, de ellos 37 pacientes eran TB-MDR y 111 TB sensible, de los cuales 52 historias fueron excluidas del estudio por datos incompletos necesarios para la investigación.

\section{Procedimientos y variables}

Las evaluaciones clínicas, radiográficas y de baciloscopía se realizaron al iniciar el tratamiento y posteriormente de manera mensual hasta culminar el tratamiento, en los servicios del Programa de Control de Tuberculosis (PCT) de cada uno de los establecimientos incluidos en el estudio, que contaban con un médico neumólogo 
o infectólogo, una licenciada en enfermería, y personal técnico; y donde los pacientes con tuberculosis pulmonar, tanto sensibles como MDR, recibieron el tratamiento específico bajo observación directa de la toma.

Se definió como tuberculosis MDR (caso) a todos los pacientes que tuviesen pruebas rápidas $\mathrm{y} / \mathrm{o}$ convencionales con resultado de resistencia, y como tuberculosis sensible (controles) a todo paciente diagnosticado por pruebas rápidas 0 pruebas convencionales como TB sensible, o aquellos que estaban recibiendo tratamiento antituberculoso para TB sensible y presentaban adecuada evolución clínica.

Se realizó muestreo de tipo no probabilístico con todos los pacientes diagnosticados con tuberculosis.

Los datos fueron obtenidos por la revisión de historias clínicas, empleándose una ficha de recolección, el cual fue validado por juicio de expertos.

\section{Análisis estadístico}

El procesamiento de datos se realizó usando el software SPSS v. 15.0, utilizándose Test de Fisher para las variables diabetes mellitus, uso de drogas y fiebre persistente a las dos semanas de tratamiento; el test de $U$ de Mann Whitney para la variable edad y para las variables cualitativas se empleó la prueba estadística de chi cuadrado.

Se realizó análisis multivariado con las variables que obtuvieron significancia estadística en el análisis bivariado. Se construyó un modelo predictivo empleando la regresión logística multivariada con prueba de bondad de ajuste para evaluar la predictividad. En la tabla generada se presentan solo las variables que mantiene la significancia estadística, además de considerar a la edad y sexo como variables confusoras y controlando el sesgo.

\section{Consideraciones éticas}

Los datos fueron confidenciales protegiéndose la identidad de los pacientes mediante asignación de código único y se respetaron los principios éticos de la Declaración de Helsinski de las Asociación Médica Mundial. El protocolo de investigación, antes de su ejecución, fue revisado y aprobado por el comité de ética en investigación de la Facultad de Medicina de la Universidad Nacional Hermilio Valdizán de Huánuco.

\section{RESULTADOS}

Durante los años del 2010 al 2015 en los cuatro establecimientos de salud que fueron incluidos en el estudio, se registraron un total de 200 casos de tuberculosis pulmonar, encontrándose un 90\% de historias clínicas completas y con adecuado seguimiento. Se incluyeron 37 casos que fueron diagnosticados como TB-MDR, a los cuales se asignó 111 controles, cuyas características epidemiológicas se encuentran en la Tabla 1.

Enelanálisisbivariadoseencontróquelaasociaciónentre la TB-MDR y el uso de alcohol resultó estadísticamente significativo, pero no con el uso de drogas ni tabaco. La presencia de contactos con tuberculosis en los pacientes con tuberculosis pulmonar fue de sólo un $1 \%$ en los controles y de $0 \%$ en los casos de pacientes TB-MDR, no encontrándose asociación alguna. Se encontró asociación significativa de la regularidad del tratamiento de pacientes con TB sensible, como factor protector para el desarrollo de la TB-MDR.

Los patrones radiográficos más frecuentes al diagnóstico de pacientes con TB-MDR fueron el patrón cavitario y el reticular, evidenciándose una asociación estadísticamente significativa con el patrón radiográfico cavitario. La relación entre la TB-MDR y la baciloscopía positiva al diagnóstico y al primer mes de tratamiento resultó estadísticamente significativa, el resto del análisis puede observarse en la Tabla 2.

En el análisis multivariado que se muestra en la Tabla 3 resultó estadísticamente significativa la asociación de la TB-MDR con la fiebre persistente, el patrón radiográfico cavitario, la baciloscopía positiva al primer mes de tratamiento, tos productiva al diagnóstico y los crépitos pulmonares al diagnóstico. 
Tabla 1. Características epidemiológicas de los pacientes con tuberculosis pulmonar en la ciudad de Huánuco del 2010 al $2015(n=148)$.

\section{Características}

Hospital / establecimiento de Salud

Centro de Salud Aparicio Pomares

61

50

Centro de Salud Las Moras

Hospital Regional Hermilio Valdizán Medrano

22

15

Hospital II EsSalud

\section{Año de historia clínica}

2010

2011

2012

2013

2014

2015

Género

Femenino

Masculino

\section{Nivel educativo}

No escolarizado

8

$5,4 \%$

Primaria

29

Secundaria

82

29

$8,1 \%$

25

$16,9 \%$

$16,2 \%$

24

30

36

21

$20,3 \%$

$24,3 \%$

$14,2 \%$

Superior

Estado civil

Casado (a)

Soltero (a)

Viudo (a)

\section{Ocupación}

Estudiante

No profesional de salud

Profesional de Salud

Sin ocupación

\section{Lugar de procedencia (distrito/ provincia)}

Amarilis (Huánuco)

Ambo (Ambo)

Chinchao (Huánuco)

Huánuco (Huánuco)

Panao (Pachitea)

Pillcomarca (Huánuco)

1

$0,7 \%$

Rondos (Lauricocha)

\section{Edad (años)}

(X+DS) 
Tabla 2. Análisis bivariado de TB-MDR en pacientes con tuberculosis pulmonar de la ciudad de Huánuco del 2010 al $2015(n=148)$.

\begin{tabular}{|c|c|c|c|c|c|c|c|c|c|}
\hline \multirow[b]{2}{*}{ Características } & \multicolumn{4}{|c|}{ Tuberculosis } & \multirow[b]{2}{*}{$\mathbf{X} 2$} & \multirow[b]{2}{*}{$\mathbf{p}$} & \multirow[b]{2}{*}{ OR } & \multicolumn{2}{|c|}{ IC $95 \%$} \\
\hline & $\begin{array}{l}\text { MDR } \\
n=37\end{array}$ & $\%$ & $\begin{array}{c}\text { Sensible } \\
n=111\end{array}$ & $\%$ & & & & Inf & Sup \\
\hline \multicolumn{10}{|l|}{ Estado civil } \\
\hline No soltero & 14 & 40,5 & 22 & 19,8 & 4,89 & 0,027 & 2,46 & 1,09 & 5,55 \\
\hline Soltero & 23 & 59,5 & 89 & 80,2 & & & & & \\
\hline \multicolumn{10}{|l|}{ Consumo de alcohol } \\
\hline $\mathrm{Si}$ & 16 & 43,2 & 19 & 17,1 & 10,49 & 0,003 & 3,69 & 1,63 & 8,35 \\
\hline No & 21 & 56,8 & 92 & 82,9 & & & & & \\
\hline \multicolumn{10}{|l|}{ Diabetes Mellitus } \\
\hline $\mathrm{Si}$ & 4 & 10,8 & 1 & 0,9 & 5,59 & $0,014 *$ & 13,33 & 1,44 & 123,45 \\
\hline No & 33 & 89,2 & 110 & 99,1 & & & & & \\
\hline \multicolumn{10}{|c|}{ Regularidad de tratamiento } \\
\hline sí & 27 & 73,0 & 101 & 91,0 & 6,244 & 0,0125 & 0,27 & 0,10 & 0,71 \\
\hline No & 10 & 37,0 & 10 & 9,0 & & & & & \\
\hline
\end{tabular}

\section{Tos productiva al diagnóstico}

$\begin{array}{lccccccccc}\text { Sí } & 31 & 83,8 & 48 & 43,2 & 16,73 & 0,000 & 6,78 & 2,62 & 17,56 \\ \text { No } & 6 & 16,2 & 63 & 56,8 & & & & & \\ \text { Crepitantess al diagnóstico } & & & & & & & & & \\ \text { Sí } & 11 & 29,7 & 9 & 8,1 & 9,32 & 0,002 & 4,79 & 1,79 & 12,78 \\ \text { No } & 26 & 70,3 & 102 & 91,9 & & & & & \end{array}$

Fiebre a las 2 semanas de tratamiento

$\begin{array}{lrrrrrrrrr}\text { Sí } & 5 & 9,0 & 1 & 1,4 & 8,34 & 0,004^{*} & 17,19 & 1,94 & 152,48 \\ \text { No } & 32 & 91,0 & 110 & 98,6 & & & & & \end{array}$

\section{Patrón radiográfico al diagnóstico}

\begin{tabular}{lccccccccc} 
Patrón cavitario & 22 & 59,5 & 13 & 11,7 & 35,04 & 0,000 & 11,06 & 4,60 & 26,52 \\
No patrón cavitario & 15 & 40,5 & 98 & 88,3 & & & & & \\
$\begin{array}{l}\text { Baciloscopía al primer mes de tratamiento } \\
\text { Positivo }\end{array}$ & & & & & & & & \\
Negativo & 14 & 52,9 & 8 & 7,7 & 47,35 & 0,000 & 21,15 & 7,94 & 56,31 \\
Edad (X + DS) & $34,05+14,45$ & $29,78+16,67$ & $1531,00 \#$ & 0,021 & & \\
IMC al diagnóstico (X + DS) & $21,74+2,37$ & $20,53+2,92$ & $1561,50 \#$ & 0,029 & & & \\
\hline
\end{tabular}

* Test exacto de Fisher / \# U de Mann Whitney. 
Tabla 3. Regresión logística de la TB MDR con factores predictores en pacientes con tuberculosis pulmonar de la ciudad de Huánuco del 2010 al 2015 ( $n=148)$.

\begin{tabular}{lccccc} 
& Z & Paracterística & OR & \multicolumn{3}{c}{ IC 95\% } \\
\hline Género & $-0,04$ & 0,969 & 0,97 & 0,3 & 3,4 \\
\hline Edad & 0,68 & 0,741 & 1,0 & 1,0 & 1,1 \\
\hline Patrón radiográfico cavitario & 3,90 & 0,000 & 18,02 & 4,2 & 77,1 \\
\hline Fiebre a las 2 semanas & 2,50 & 0,012 & 61,8 & 2,4 & 1570,0 \\
\hline Baciloscopía positiva al primer mes de tratamiento & 4,85 & 0,000 & 47,4 & 9,9 & 225,8 \\
\hline Tos productiva al diagnóstico & 2,43 & 0,015 & 5,48 & 1,4 & 21,6 \\
\hline Crépitos al diagnóstico & 2,38 & 0,017 & 8,4 & 1,4 & 48,1 \\
\hline
\end{tabular}

Prueba de bondad de ajuste:

Hosmer-Lemeshow chi2 $(10)=3,49$

Prob $>$ chi $2=0,9675$

\section{DISCUSIÓN}

La tuberculosis es un enorme problema de salud pública a nivel mundial y en el Perú, a pesar de una reducción de su incidencia en los últimos años, aun afecta principalmente a la población con menos recursos económicos ${ }^{(2)}$.

Uno de los objetivos de nuestro estudio fue encontrar la asociación entre TB-MDR y la fiebre persistente, definida como aquella que duraba más de 2 semanas después del inicio del tratamiento con medicamentos de primera línea (isoniacida, rifampicina, pirazinamida, etambutol); resultando estadísticamente significativo, lo cual significa que la persistencia de la fiebre a pesar de haber iniciado tratamiento con fármacos antituberculosos predice la presencia de TB-MDR. Esto es semejante a lo encontrado por Solomon y cols, quienes al evaluar la respuesta de la fiebre a la terapia antituberculosa, hallaron que la disminución o desaparición de la fiebre fue menos probable en los pacientes que tenían TBMDR, que en aquellos pacientes con tuberculosis sensible ${ }^{(7)}$.

La radiografía de tórax es considerada un pilar en el diagnóstico de tuberculosis pulmonar, ya que una radiografía de tórax normal disminuye la probabilidad de la existencia de una enfermedad activa. La tuberculosis pulmonar se puede manifestar con diferentes patrones radiológicos, dependiendo del grupo de edad, el estado inmunológico del paciente, entre otros factores. El patrón cavitario se produce cuando el material caseoso licuefaccionado se expulsa desde el centro de la lesión hacia el interior del árbol bronquial, puede variar en su tamaño, y puede ser única o múltiple. En nuestro estudio, se encontró asociación significativa de TB-MDR con el patrón radiográfico cavitario. En países con alta prevalencia e incidencia de tuberculosis, se han realizado estudios con el objetivo de buscar la asociación entre TB-MDR y diferentes patrones radiográficos. Chuchottaworn y cols realizaron un estudio en Tailandia, para determinar los factores de riesgo asociados a TB-MDR, encontrando, al igual que nosotros, asociación con la presencia de cavidades en la radiografía de tórax; adicionalmente, hallaron significancia estadística con ciertas características particulares de las cavidades como: el número de cavidades de 3 a más, el diámetro máximo mayor a $30 \mathrm{~mm}$ y más de 2 zonas pulmonares afectadas ${ }^{(11)}$. Lai y cols en 2010, hallaron que los pacientes con TB-XDR (extremadamente resistente) tenían una significativa mayor prevalencia de lesiones pulmonares cavitarias en la radiografía de tórax, que los pacientes con TB-MDR $(p<0,05)^{(12)}$, sugiriendo que la presencia de patrón cavitario está asociado a mayor resistencia.

Un sencillo predictor de laboratorio, que se obtiene de manera rutinaria en todo paciente con diagnóstico con tuberculosis pulmonar, es la baciloscopía. En el caso de nuestro estudio se evidencia la clara asociación entre la baciloscopía persistentemente positiva al primer mes de tratamiento con el posterior diagnóstico de TB-MDR; que coincide con los resultados obtenidos en una investigación peruana en la que se concluye que, de 1545 pacientes estudiados, 145 de ellos tenían baciloscopía positiva durante los primeros 60 días de tratamiento, y el $21,8 \%$ de estos pacientes tenían 
algún tipo de resistencia ${ }^{(13)}$. Contrariamente, un estudio realizado por Kumar y cols encontró baciloscopía positiva a los dos, cuatro, cinco y seis meses después del inicio del tratamiento, sin embargo, al realizar cultivos de estos pacientes resultaron negativos en más del $60 \%$, concluyendo que el iniciar tratamiento empírico para TB-MDR en base a las baciloscopías persistentemente positivas es incorrecto y tendría peligrosas consecuencias ${ }^{(14)}$.

Nuestro estudio no encontró asociación entre el sexo y la posibilidad de desarrollar TB-MDR, semejante a una investigación realizada en Perú, donde a pesar de haber mayor frecuencia de sexo masculino en el grupo de controles, al realizar el análisis estadístico, no existían diferencias significativas ${ }^{(15)}$; sin embargo, una investigación realizada en China mostró que el sexo masculino tuvo mayor probabilidad de desarrollar TBMDR que el sexo femenino (8,3\% vs. 3,3\%; $X 2=8,69, p$ $<0,001)^{(16)}$.

Los grupos de edad en los que puede presentarse la TBMDR son variados y dependientes de diversos factores. En nuestro estudio, la media de edad para los casos fue de 33,12 + 11,66 años, y para los controles, de 29,82+ 16,69 años, sin llegar a ser estadísticamente significativo. Estos hallazgos coinciden con los resultados del estudio de Mekonnen, quien evaluó la prevalencia y factores de riesgo de TB-MDR en Etiopía, donde la media y mediana de edad fue de 32 y 29 años respectivamente, pero ningún grupo de edad resultó estadísticamente significativo ${ }^{(17)}$. Contrariamente, algunas investigaciones han encontrado asociación estadísticamente significativa entre grupos de edad específicos y el riesgo de desarrollar TB-MDR, sin embargo, los resultados de los estudios no son uniformes, porque difieren en el grupo de edad. Por ejemplo, Ávalos-Rodríguez y cols estudiaron los factores asociados a la TB-MDR primaria en el Callao, Perú, encontrando que una edad mayor a 40 años resultó ser un factor protector frente a la TB-MDR, es decir, el mayor riesgo se encontró en los pacientes más jóvenes ${ }^{(18)}$. Por otro lado, el grupo de Zhao, desarrolló un metaanálisis en China, encontrando asociación estadísticamente significativa con un grupo de mayor edad, entre los 40 y 60 años $^{(19)}$.

El presente estudio consideró tres factores sociales asociados a la tuberculosis pulmonar: el uso de alcohol, tabaco y drogas. La frecuencia de uso de estos fue baja tanto en los pacientes con TB-MDR como sensibles. En el análisis multivariado, no se encontró asociación estadísticamente significativa con el uso del tabaco ni de drogas, pero si se evidenció asociación con el uso de alcohol. De manera similar, Odone y cols. realizaron un estudio de cohortes en Lima, acerca del rol de los determinantes sociales en la tuberculosis
MDR, que incluyó dentro de sus variables estudiadas el uso de tabaco $y$, al igual que nuestro estudio, no encontró asociación significativa con haber sido o ser actualmente fumador(20); igualmente el grupo de Martínez, realizó un estudio de cohorte prospectivo en 37 centros de salud localizados en áreas pobres de Lima, encontrando significativa la asociación entre la TB-MDR y el uso de alcoholl(11). Con respecto al uso de drogas, otras investigaciones encuentran asociación de la TB-MDR con el uso de drogas, pero solo con algunos tipos de drogas. Young en su estudio acerca de los predictores sociales y clínicos de tuberculosis resistente en Monterrey, México, encontró asociación significativa de la TB resistente (que incluía a la TB-MDR) con el uso de cocaína, pero no con el uso de marihuana, metanfetamina ni drogas intravenosas ${ }^{(21)}$.

Las comorbilidades que se evaluaron en nuestro estudio fueron la infección por el VIH y la diabetes mellitus. En cuanto al $\mathrm{VIH}$, no se encontró ningún paciente seropositivo dentro de la muestra, mientras que la frecuencia de la diabetes mellitus en los pacientes con TB sensible fue de sólo el 0,9\%, y de 10,8\% en los pacientes con TB-MDR, datos que resultaron en una asociación estadísticamente significativa en el análisis bivariado, pero debido a la muestra tan escasa requiere mayores estudios al respecto, puesto que un estudio realizado en el 2014, evidencia que la diabetes mellitus en el análisis multivariado no estuvo asociado significativamente a ninguna resistencia a los medicamentos ${ }^{(22)}$.

El contacto con pacientes con tuberculosis es considerado según la guía del Ministerio de Salud del Perú como factor de riesgo para TB-MDR; el cual se encuentra avalado por algunas investigaciones como un metaanalisis desarrollado en Nueva York en el cual, se encontraron 25 estudios que evaluaron una mediana de 111 contactos familiares de pacientes farmacorresistentes, el rendimiento combinado fue de 7,8\% (IC del 95\%, 5,6\% -10,0\%) para la tuberculosis activa y 47,2\% (IC del 95\%, 30,0\% -61,4\%) para la tuberculosis latente, aunque hubo heterogeneidad estadística significativa $(P<0,0001)$, dicho estudio concluye que la investigación de contactos de los hogares en torno a la resistencia a los medicamentos antituberculosis es una intervención de alto rendimiento para la detección de la tuberculosis y la prevención de TB-MDR ${ }^{(23)}$, contrario a los hallazgos de nuestro estudio, en que no se encontró asociación significativa, tal vez debido al escaso número de contactos con pacientes con TB sensible y a la nula cantidad de contactos con pacientes TB-MDR; dicha evidencia también se observa en un estudio realizado en Tailandia, en la que de manera similar al nuestro, no se evidencia asociación estadística(24).

Nuestro estudio identificó que la regularidad y el 
cumplimiento del tratamiento en pacientes con TB sensible es un factor protector frente a la TB-MDR. La irregularidad al tratamiento es considerada por muchas investigaciones como un factor de riesgo para TB-MDR, así lo demuestra un metaanálisis realizado en China, que al evaluar la mala adherencia al tratamiento, encontró que fue un factor de riesgo para desarrollar TB-MDR ${ }^{(19)}$.

\section{LIMITACIONES}

Encontramos que durante el desarrollo de nuestro estudio la limitación más importante fue la fuente de información utilizada, puesto que un porcentaje de las historias clínicas utilizadas se encontraron incompletas, por lo que se excluyeron de la investigación resultando en un menor número de pacientes para el estudio. Además, nuestro diseño al ser retrospectivo, presenta sesgos propios de este tipo de investigación, por lo que su extrapolación puede ser limitada.

\section{CONCLUSIÓN}

En nuestro estudio los factores predictores asociados en forma independiente a la TB-MDR en pacientes con tuberculosis pulmonar fueron la fiebre persistente a las dos semanas de iniciado el tratamiento, la tos productiva al momento del diagnóstico, los crepitantes pulmonares al diagnóstico, el patrón radiográfico cavitario y la baciloscopía persistentemente positiva al primer mes de tratamiento.

Contribuciones de autoría: Los autores participaron en la génesis de la idea, diseño de proyecto, recolección e interpretación de datos, análisis de resultados y preparación del manuscrito del presente trabajo de investigación.

\section{Financiamiento: Autofinanciado.}

Conflicto de interés: Los autores declaran no tener conflicto de interés en la publicación de este artículo.

Recibido: 22 de enero 2020

Aprobado: 10 de marzo 2020

\section{Correspondencia: Kovy Arteaga-Livias.}

Dirección: Av. Universitaria N 601-607, Pillco Marca 10003, Huánuco-Perú. Teléfono: +51987835517

Correo:farteaga@unheval.edu.pe

\section{REFERENCIAS BIBLIOGRÁFICAS}

1. Organización Mundial de la Salud. Tuberculosis. Nota descriptiva №104. Octubre 2016

2. Organización Panamericana de la Salud. La tuberculosis en la Región de las Américas: Informe Regional 2012. Epidemiología, control y financiamiento. Washington, DC: OPS, 2014.

3. Del Castillo H., Mendoza A., Saravia J., Somocurcio J. Epidemia de tuberculosis multidrogoresistente y extensivamente resistente a drogas en el Perú: Situación y propuestas para su control. Rev Peru Med Exp Salud Pública. 2009; 26(3): 380-86.

4. World Health Organization [base de datos en Internet]. Diagnosis and notification of resistant TB 2014 [acceso 01 de mayo de 2016]. Disponible en:https://extranet.who.int/sree/Reports?op=vs\&path=\%2FWHO_ HQ_Reports\%2F G $2 \% 2$ F P R D \% 2 FEXT\%2 FDRTB_ map\&propWidth=1366\&propHeight $=643$.

5. Oficina de informática, telecomunicaciones y estadística - DIRESAHCO. Consolidado anual programa PCT a nivel de provincias, distritos, y establecimientos de salud. 2014.

6. Martínez D, Heudebert G, Seas C, Henostroza G, Rodriguez M, Zamudio C, et al. Clinical Prediction Rule for Stratifying Risk of Pulmonary MultidrugResistant Tuberculosis. Plos One. 2010. 5(8): e12082.

7. Salomon N, Perlman DC, Friedmann P, Buchstein S, Kreiswirth BN, Mildvan D. Predictors and Outcome of Multidrug - Resistant Tuberculosis. Clin Res Infect Dis. 1995.21:1245-52.

8. Otu A, Umoh V, Habib A, Ansa V. Prevalence and clinical predictors of drugresistant tuberculosis in three clinical settings in Calabar, Nigeria. Clin Respir 2014; 8: 234-239.

9. Crispín V, Roque M, Salazar EM, Ruiz RJ, Ortiz J, Almonacid A, et al. Factores de Riesgo para tuberculosis multidrogoresistente en Establecimientos de Salud Urbano Marginales 2006 - 2008. Cienc Invest. 2012; 15(1): 25-29.

10. Álvarez GGC, Sandoval TH, Bojalil JLF. Tuberculosis resistente al tratamiento por fármacos antifímicos. Estudio en el Estado de Chiapas, México. Aten Primaria 1999; 24:209-14.

11. Chuchottaworn C, Thanachartwet V, Sangsayunh P, Myint TZ, Sahassananda D, Surabotsophon M, et al. Risk Factors for Multidrug-Resistant Tuberculosis among Patients with Pulmonary Tuberculosis at the Central Chest Institute of Thailand. Plos One.2015; 10(10):e0139986.

12. Lai CC, Tan CK, Lin SH, Liao CH, Huang YT, Chou CH, et al. Clinical and genotypic characteristics of extensively drug-resistant and multidrug-resistan tuberculosis. Eur J Clin Microbiol Infect Dis.2010; 29:597-600.

13. Laura J. Martin, Martha H. Roper, Louis Grandjean. Robert H. Gilman, et al. Rationing tests for drug-resistant tuberculosis - who are we prepared to miss? BMC Medicine (2016); 14:30.

14. Kumar $M$, Claassens V, Banurekha V. Are we justified in treating for multidrug-resistant tuberculosis based on positive follow-up smear results? Cape Town, South Africa. Int J Tuberc Lung Dis 18(4): 449-453.

15. Rodríguez Hidalgo, Luís Alejandro. Factores de riesgo para tuberculosis pulmonar multidrogoresistente en la región La Libertad, Perú. Sciendo. 2012; 15(2):45-54.

16. Yang $Y$, Zhou C, Shi L, Meng H, Yan H. Prevalence and characterization of drug-resistant tuberculosis in a local hospital of Northeast China. Int J Infect Dis. 2014 May;22:83-6.

17. Mekonnen F, Tessema B, Moges F, Gelaw A, Eshetie S, Kumera G. Multidrug resistant tuberculosis: prevalence and risk factors in districts of metema and west armachiho, Northwest Ethiopia. BMC Infectious Diseases.2015; 15:461.

18. Avalos A, Imán F, Virú M, Cabrera J, Zárate $A$, Meza $M$, et al. Factores asociados a tuberculosis MDR primaria en pacientes de Callao, Perú. An Fac med. 2014; 75(3):233-6.

19. Zhao P, Xj L, Zhang S, Wang K and Liu C. Social Behaviour Risk Factors for Drug Resistant Tuberculosis in Mainland China: a Meta-analysis. China. J Int Med Res. 2012; 40(2):436-45

20. Odone A, Calderon R, Becerra MC, Zhang Z, Contreras CC, Yataco R, et al. Acquired and Transmitted Multidrug Resistant Tuberculosis: The Role of Social Determinants. Plos One. 2015; 11(1): e0146642.

21. Young B, Burgos M, Handal A, Baker J, Rendón A, et al. Social and clinical predictors of drug-resistant tuberculosis in a public hospital, Monterrey, Mexico. Annals of Epidemiology. 2014; 24:771 -75

22. Nhamoyebonde S, Leslie A. Biological Differences Between the Sexes and Susceptibility to Tuberculosis. Durban, South Africa. J Infect Dis 2014:209 (Suppl 3). S100-S106.

23. Grandjean L, Crossa A, Gilman R, Herrera C. Tuberculosis in household contacts of multidrug-resistant tuberculosis patients. Int J Tuberc Lung Dis 15(9):1164-1169.

24. Shah NS, Yuen CM, Heo M, Tolman AW, Becerra MC. Yield of contact investigations in households of patients with drug-resistant tuberculosis: systematic review and meta-analysis. Clin Infect Dis. 2014: 58(3):381-91. 